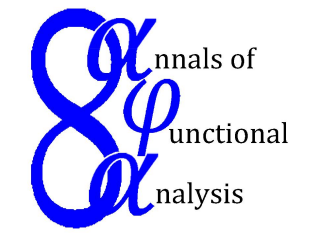

Ann. Funct. Anal. 5 (2014), no. 2, 138-146

$\mathscr{A}$ NNALS OF $\mathscr{F}$ UNCTIONAL $\mathscr{A}$ NALYSIS

ISSN: 2008-8752 (electronic)

URL:www.emis.de/journals/AFA/

\title{
A NORMAL VARIATION OF THE HORN PROBLEM: THE RANK 1 CASE
}

\author{
LEI CAO* AND HUGO J. WOERDEMAN \\ Dedicated to Professor Tsuyoshi Ando, \\ a valued colleague and friend, with admiration and respect
}

Communicated by M. S. Moslehian

\begin{abstract}
Given three $n$-tuples $\left\{\lambda_{i}\right\}_{i=1}^{n},\left\{\mu_{i}\right\}_{i=1}^{n},\left\{\nu_{i}\right\}_{i=1}^{n}$ of complex numbers, we introduce the problem of when there exists a pair of normal matrices $A$ and $B$ such that $\sigma(A)=\left\{\lambda_{i}\right\}_{i=1}^{n}, \sigma(B)=\left\{\mu_{i}\right\}_{i=1}^{n}$, and $\sigma(A+B)=\left\{\nu_{i}\right\}_{i=1}^{n}$, where $\sigma(\cdot)$ denote the spectrum. In the case when $\lambda_{k}=0, k=2, \ldots, n$, we provide necessary and sufficient conditions for the existence of $A$ and $B$. In addition, we show that the solution pair $(A, B)$ is unique up to unitary similarity. The necessary and sufficient conditions reduce to the classical A. Horn inequalities when the $n$-tuples are real.
\end{abstract}

\section{INTRODUCTION}

The classical problem of A. Horn [5] asks whether for given real numbers $\lambda_{1}, \ldots, \lambda_{n}, \mu_{1}, \ldots, \mu_{n}, \nu_{1}, \ldots, \nu_{n}$, there exist $n \times n$ Hermitian matrices $A$ and $B$ so that

$$
\sigma(A)=\left\{\lambda_{1}, \ldots, \lambda_{n}\right\} \sigma(B)=\left\{\mu_{1}, \ldots, \mu_{n}\right\} \text { and } \sigma(A+B)=\left\{\nu_{1}, \ldots, \nu_{n}\right\} .
$$

The conjectured solution by A. Horn involves the trace equality

$$
\sum_{i=1}^{n} \lambda_{i}+\sum_{i=1}^{n} \mu_{i}=\sum_{i=1}^{n} \nu_{i}
$$

and a list of inequalities on the given real numbers, termed the Horn inequalities. It took more than 30 years to resolve the Horn conjecture. In 1998, Alexander

Date: Received: November 20, 2013; Accepted: December 5, 2013.

* Corresponding author.

2010 Mathematics Subject Classification. Primary 15A18; Secondary 47B15.

Key words and phrases. The problem of A. Horn, normal matrices, upper Hessenberg. 
Klyachko [6] gave the connection between the so-called 'Saturation conjecture' and the Horn conjecture. In 1999, the Saturation Conjecture was proved by Allen Knutson and Terence Tao [7], and as a consequence the Horn conjecture was solved.

In this paper, we propose a normal variation of this problem, the Eigenvalue Problem for the Sum of Normal Matrices (EPSNM): Given complex numbers $\lambda_{1}, \ldots, \lambda_{n}, \mu_{1}, \ldots, \mu_{n}$, and $\nu_{1}, \ldots, \nu_{n}$, when do there exist $n \times n$ normal matrices $A$ and $B$ so that (1.1) holds?

As the solution to the Horn conjecture involved techniques from representation theory, combinatorics, and geometric invariant theory, a solution to EPSNM is expected to require substantial machinery outside of linear algebra as well. A notable difference with the Hermitian case is the fact that the sum of two normal matrices is in general not normal. Generalizing results from the Hermitian case to the normal often provide new challenges; see for instance [1]. However, as observed in [2], the Horn problem for the special case where $A$ has rank 1 can be solved with linear algebra techniques. Using these ideas, we are able to give a solution to EPSNM when $\lambda_{2}=\ldots=\lambda_{n}=0$. We also show that in this case the solution pairs $(A, B)$ are unique up to unitary similarity. Our main result is the following.

Theorem 1.1. Let $\lambda=\left(\lambda_{1}, 0, \ldots, 0\right), \mu=\left(\mu_{1}, \mu_{2}, \ldots, \mu_{n}\right), \nu=\left(\nu_{1}, \nu_{2}, \ldots, \nu_{n}\right) \in$ $\mathbb{C}^{n}$. There exists normal matrices $A, B$ such that

$$
\sigma(A)=\lambda, \quad \sigma(B)=\mu, \quad \sigma(A+B)=\nu
$$

if and only if the trace equality (1.2) holds, and the rational function

$$
\Delta(x):=\frac{\prod_{i=1}^{n}\left(x-\nu_{i}\right)}{\lambda_{1} \prod_{i=1}^{n}\left(x-\mu_{i}\right)} \text { only has simple poles }
$$

and satisfies

$$
\operatorname{Res}_{\mu_{k}}(\Delta(x)) \leq 0 \text { for } k=1,2, \ldots, n .
$$

Moreover, if $(\tilde{A}, \tilde{B})$ is another solution, then there exists a unitary matrix $U$, so that

$$
A=U \tilde{A} U^{*}, \quad B=U \tilde{B} U^{*} .
$$

Here $\operatorname{Res}_{\mu_{k}}(\Delta(x))$ is the residue of $\Delta(x)$ at $\mu_{k}$. When $\mu_{1}, \ldots, \mu_{n}$ are all different, conditions (1.4) and (1.5) reduce to

$$
\frac{\prod_{i=1}^{n}\left(\mu_{k}-\nu_{i}\right)}{\lambda_{1} \prod_{i=1, i \neq k}^{n}\left(\mu_{k}-\mu_{i}\right)} \leq 0 \text { for } k=1,2, \ldots, n .
$$

In general the problem can be reduced to the case of different $\mu$ 's, as when $\mu_{i}=\mu_{k}$ for some $i \neq k$ we must have that $\nu_{j}=\mu_{i}$ for some $j$. Removing a 0 from $\lambda$, $\mu_{i}$ from $\mu$, and $\nu_{j}$ from $\nu$, one obtains a problem for $(n-1) \times(n-1)$ matrices whose solution gives a solution to the original problem (by direct summing). By repeating this reduction until all $\mu$ 's are different, one can state the the necessary and sufficient conditions as inequalities of the form (1.6).

In the proof of Theorem 1.1 we make use of results in [10] where the classical Cauchy-Poincaré interlacing inequalities were generalized to the setting of normal 
matrices; see Proposition 2.4. In addition, in [10] a uniqueness result for upper Hessenberg normal matrices was stated, a normal variation of a result in [4] concerning Hermitian tridiagonal matrices; see Theorem 2.5. This latter result is used to prove the uniqueness statement in Theorem 1.1. We end the introduction with noting that Wielandt [11] characterized when a complex number $\nu$ is the eigenvalue of the sum of two normal matrices with prescribed eigenvalues. This was further pursued in [9].

\section{Proof of the MAIN Result}

Definition 2.1. A matrix $A=\left(a_{i j}\right)_{i, j=1}^{n}$ is called upper Hessenberg if $a_{i j}=0$ for all $i \geq j+2$. An upper Hessenberg matrix is called unreduced if all $a_{i+1, i} \neq 0$, for $i=1,2, \ldots, n-1$, and positively unreduced if $a_{i+1,1}>0$, for $i=1,2, \ldots, n-1$.

Using standard Householder techniques, one can easily put any matrix in upper Hessenberg form (see, e.g, [3], Section 7.4, Algorithm 7.4.2), which is the content of the following auxiliary result.

Lemma 2.2. If $A$ is an $n \times n$ matrix, then there exists a unitary matrix $P$ of the form

$$
\left(\begin{array}{cccc}
1 & 0 & \ldots & 0 \\
0 & * & \ldots & * \\
\vdots & \vdots & \ddots & \vdots \\
0 & * & \ldots & *
\end{array}\right)
$$

such that $\hat{A}=P A P^{*}$ is upper Hessenberg.

For a matrix $B$, we denote its characteristic polynomial by $P_{B}$ (that is, $P_{B}(x)=$ $\operatorname{det}(x I-B))$ and the submatrix of $B$ obtained by removing row $i$ and column $j$ is denoted by $B(i \mid j)$.

Lemma 2.3. Let

$$
A=\left(\begin{array}{cccc}
\lambda_{1} & & & \\
& 0 & & \\
& & \ddots & \\
& & & 0
\end{array}\right)
$$

and suppose $\mu=\left(\mu_{1}, \ldots, \mu_{n}\right), \nu=\left(\nu_{1}, \ldots, \nu_{n}\right)$ and $\tau=\left(\tau_{1}, \ldots, \tau_{n-1}\right)$ are complex tuples and there exists a normal matrix $B$ such that

$$
\sigma(B)=\mu, \sigma(A+B)=\nu \text { and } \sigma(B(1 \mid 1))=\tau .
$$

If $\mu_{i}$ is an eigenvalue of $B$ of geometric (or, equivalently, algebraic) multiplicity $m$, then

i. $\mu_{i}$ is an eigenvalue of $A+B$ of geometric multiplicity at least $m-1$;

ii. $\mu_{i}$ is an eigenvalue of $B(1 \mid 1)$ of algebraic multiplicity at least $m-1$.

Proof. The rank of $A$ is 1 . Since $\mu_{i}$ is an eigenvalue of $B$ of geometric multiplicity $m$, the rank of $\mu_{i} I-B$ is $n-m$. Then the rank of $\mu_{i} I-(A+B)$ is at most 
$n-m+1$ meaning that $\mu_{i}$ is an eigenvalue of $A+B$ of geometric multiplicity at least $m-1$. Since

$$
P_{B(1 \mid 1)}(x)=\frac{1}{\lambda_{1}}\left(P_{B}(x)-P_{A+B}(x)\right)
$$

and both $P_{B}(x)$ and $P_{A+B}(x)$ have $\mu_{i}$ as a root of multiplicity at least $m-1$, $P_{B(1 \mid 1)}(x)$ has $\mu_{i}$ as a root of multiplicity at least $m-1$.

We recall the following results from [10].

Proposition 2.4 ([10], Proposition 3.1). Let $\left\{\mu_{i}\right\}_{1}^{n}$ and $\left\{\tau_{j}\right\}_{1}^{n-1}$ be two sequences of complex numbers. There exists a normal matrix $B$ such that

$$
\sigma(B)=\left\{\mu_{1}, \mu_{2}, \ldots, \mu_{n}\right\} \text { and } \sigma(B(1 \mid 1))=\left\{\tau_{1}, \ldots, \tau_{n-1}\right\}
$$

if and only if

$$
\Lambda(x)=\frac{\prod_{i=1}^{n-1}\left(x-\tau_{i}\right)}{\prod_{i=1}^{n}\left(x-\mu_{i}\right)} \text { only has simple poles }
$$

and

$$
\operatorname{Res}_{\mu_{k}}(\Lambda(x)) \geq 0 \text { for } k=1,2, \ldots, n .
$$

Theorem 2.5 ([10], Corrected version of Theorem 3.7). For any $n$ distinct complex numbers $\mu_{1}, \mu_{2}, \ldots, \mu_{n}$ and any $n-1$ complex numbers $\tau_{1}, \ldots, \tau_{n-1}$ satisfying (2.2) and (2.3), there exists a unique positively unreduced normal upper Hessenberg matrix $B$, such that

$$
\sigma(B)=\left\{\mu_{i}\right\}_{1}^{n} \text { and } \sigma(B(1 \mid 1))=\left\{\tau_{i}\right\}_{1}^{n-1} .
$$

In [10] the statement allowed the possibility of some $\mu^{\prime}$ s appearing more than once, in which case one obtains a direct sum and uniqueness can no longer be guaranteed. We therefore restated the result as above. Notice that $\tau_{1}, \ldots, \tau_{n-1}$, the prescribed eigenvalues of $B(1 \mid 1)$, need not be distinct as the following example shows.

Example 2.6. Let $\mu_{1}=2, \mu_{2}=\frac{1}{2}+\frac{\sqrt{3}}{2} i, \mu_{3}=\frac{1}{2}-\frac{\sqrt{3}}{2} i$, and $\tau_{1}=\tau_{2}=1$. One can check they satisfy both (2.2) and (2.3). Then the unique positively upper Hessenberg normal matrix determined by $\mu^{\prime}$ s and $\tau^{\prime}$ s is

$$
B=\left(\begin{array}{lll}
1 & 0 & 1 \\
1 & 1 & 0 \\
0 & 1 & 1
\end{array}\right)
$$

Note that $B(1 \mid 1)$ is not normal.

Lemma 2.7. The matrix $B=\left(\begin{array}{ll}P & R \\ 0 & Q\end{array}\right)$, with $P$ and $Q$ square matrices, is normal if and only if $R=0$ and $P$ and $Q$ are normal.

Proof. The if direction is trivial, so let us assume that $B$ is normal. Then

$$
\left(\begin{array}{cc}
P & R \\
0 & Q
\end{array}\right)\left(\begin{array}{cc}
P^{*} & 0 \\
R^{*} & Q^{*}
\end{array}\right)=\left(\begin{array}{cc}
P^{*} & 0 \\
R^{*} & Q^{*}
\end{array}\right)\left(\begin{array}{cc}
P & R \\
0 & Q
\end{array}\right),
$$


and thus

$$
P P^{*}+R R^{*}=P^{*} P
$$

Taking the trace on both sides gives

$$
\operatorname{tr}\left(P P^{*}\right)+\operatorname{tr}\left(R R^{*}\right)=\operatorname{tr}\left(P^{*} P\right)=\operatorname{tr}\left(P P^{*}\right) .
$$

This implies

$$
\|R\|_{F}=0
$$

where $\|\cdot\|_{F}$ is the Frobenius norm and thus $R=0$. The normality of $P$ and $Q$ immediately follows.

Proof of Theorem 1.1. First we assume that normal matrices $A$ and $B$ exist, so that (1.1) holds. By using a unitary similarity, we may always assume that

$$
A=\left(\begin{array}{cccc}
\lambda_{1} & & & \\
& 0 & & \\
& & \ddots & \\
& & & 0
\end{array}\right) .
$$

Let $C=A+B$, it is easy to see that the corresponding characteristic polynomials $P_{B}(x), P_{C}(x)$ and $P_{B(1 \mid 1)}(x)$ satisfy

$$
P_{B(1 \mid 1)}(x)=\frac{1}{\lambda_{1}}\left(P_{B}(x)-P_{C}(x)\right) .
$$

Letting $\tau_{1}, \ldots, \tau_{n-1}$ denote the eigenvalues of $B(1 \mid 1),(2.5)$ is equivalent to

$$
\prod_{i=1}^{n-1}\left(x-\tau_{i}\right)=\frac{1}{\lambda_{1}}\left(\prod_{i=1}^{n}\left(x-\mu_{i}\right)-\prod_{i=1}^{n}\left(x-\nu_{i}\right)\right) .
$$

We get from Proposition 2.4 that $\Lambda(x)$ defined by (2.2) only has simple poles and $\operatorname{Res}_{\mu_{k}}(\Lambda(x)) \geq 0, k=1, \ldots, n$. Due to $(2.6)$,

$$
\Lambda(x)=\frac{\prod_{i=1}^{n}\left(x-\mu_{i}\right)-\prod_{i=1}^{n}\left(x-\nu_{i}\right)}{\lambda_{1} \prod_{i=1}^{n}\left(x-\mu_{i}\right)} .
$$

Now it follows directly that $\Delta(x)=\frac{1}{\lambda_{1}}-\Lambda(x)$ only has simple poles and $\operatorname{Res}_{\mu_{k}}(\Delta(x)) \leq$ 0 .

For the converse, suppose that (1.2), (2.2) and (2.3) are satisfied. Due to the trace equality (1.2), the polynomial

$$
\frac{1}{\lambda_{1}}\left(\prod_{i=1}^{n}\left(x-\mu_{i}\right)-\prod_{i=1}^{n}\left(x-\nu_{i}\right)\right)
$$

is monic of degree $n-1$; let $\tau_{1}, \ldots, \tau_{n-1}$ denote its roots. Then

$$
\prod_{i=1}^{n-1}\left(x-\tau_{i}\right)=\frac{1}{\lambda_{1}}\left(\prod_{i=1}^{n}\left(x-\mu_{i}\right)-\prod_{i=1}^{n}\left(x-\nu_{i}\right)\right) .
$$

Now $\left\{\mu_{1}, \ldots, \mu_{n}\right\}$ and $\left\{\tau_{1}, \ldots, \tau_{n-1}\right\}$ satisfy the condition of Proposition 2.4. Thus there exists a normal matrix $B$ such $\sigma(B)=\left\{\mu_{1}, \ldots, \mu_{n}\right\}$ and $\sigma(B(1 \mid 1))=$ $\left\{\tau_{1}, \ldots, \tau_{n-1}\right\}$. Let $A$ be as in (2.4). It is now easy to check that $A+B$ has the eigenvalues $\nu_{1}, \ldots, \nu_{n}$. 
Next we prove the uniqueness up to unitary similarity. We first consider the case when

$$
\left\{\mu_{1}, \mu_{2}, \ldots, \mu_{n}\right\} \cap\left\{\nu_{1}, \nu_{2}, \ldots, \nu_{n}\right\}=\emptyset
$$

which implies that all $\mu^{\prime}$ s are distinct due to Lemma 2.3. Suppose there exists two pairs of solution $A, B$ and $\tilde{A}, \tilde{B}$ with

$$
\sigma(A)=\sigma(\tilde{A})=\lambda, \sigma(B)=\sigma(\tilde{B})=\mu, \sigma(A+B)=\sigma(\tilde{A}+\tilde{B})=\nu .
$$

Then

$$
\sigma(B(1 \mid 1))=\sigma(\tilde{B}(1 \mid 1))
$$

as

$$
P_{B(1 \mid 1)}(x)=\frac{1}{\lambda_{1}}\left(\prod_{i=1}^{n}\left(x-\mu_{i}\right)-\prod_{i=1}^{n}\left(x-\nu_{i}\right)\right)=P_{\tilde{B}(1 \mid 1)}(x) .
$$

Let the roots of $P_{B(1 \mid 1)}$ be denoted by $\tau_{1}, \ldots, \tau_{n-1}$. Notice that due to $(2.9)$ we have that $\sigma(B(1 \mid 1)) \cap \sigma(B)=\emptyset$ is equivalent to

$$
\left\{\mu_{1}, \mu_{2}, \ldots, \mu_{n}\right\} \cap\left\{\nu_{1}, \nu_{2}, \ldots, \nu_{n}\right\}=\emptyset .
$$

By using unitary similarity, we may always assume

$$
A=\tilde{A}=\left(\begin{array}{cccc}
\lambda_{1} & & & \\
& 0 & & \\
& & \ddots & \\
& & & 0
\end{array}\right)
$$

So we just need to show that there exists a unitary matrix $U$ in the form $\left(\begin{array}{ll}1 & 0 \\ 0 & *\end{array}\right)$ such that $B=U \tilde{B} U^{*}$. Due to Lemma 2.2, there exists a unitary matrix $U_{1}$ in the form $\left(\begin{array}{ll}1 & 0 \\ 0 & *\end{array}\right)$ such that

$$
\hat{B}=U_{1} B U_{1}^{*}=\left(\begin{array}{ccccc}
b_{11} & \hat{b}_{12} & \ldots & \hat{b}_{1, n-1} & \hat{b}_{1 n} \\
\hat{b}_{21} & \hat{b}_{22} & \ldots & \hat{b}_{2, n-1} & \hat{b}_{2 n} \\
& \hat{b}_{32} & \ldots & \hat{b}_{3, n-1} & \hat{b}_{3 n} \\
& & \ddots & \vdots & \vdots \\
& & & \hat{b}_{n, n-1} & b_{n n}
\end{array}\right)
$$

is an upper Hessenberg matrix with $\hat{b}_{i+1, i} \geq 0$. As in addition $\sigma(\hat{B}) \cap \sigma(\hat{B}(1 \mid 1))=$ $\emptyset, \hat{B}$ is uniquely determined due to Theorem 2.5. Similarly, there exists a unitary matrix $U_{2}$ in the form $\left(\begin{array}{ll}1 & 0 \\ 0 & *\end{array}\right)$, such that $U_{2} \tilde{B} U_{2}^{*}$ is an upper Hessenburg matrix and $U_{2} \tilde{B} U_{2}^{*}$ must be $\hat{B}$ due to $(2.7),(2.8)$ and Theorem 2.5. But then it follows that $(A, B)$ and $(A, \tilde{B})$ are unitary similar.

Now suppose

$$
\left\{\mu_{1}, \mu_{2}, \ldots, \mu_{n}\right\} \cap\left\{\nu_{1}, \nu_{2}, \ldots, \nu_{n}\right\} \neq \emptyset .
$$

Let $\sigma(B(1 \mid 1))=\sigma(\tilde{B}(1 \mid 1))=\left\{\tau_{1}, \ldots, \tau_{n-1}\right\}$. Due to $(2.5)$ and (2.6),

$$
\left\{\mu_{1}, \mu_{2}, \ldots, \mu_{n}\right\} \cap\left\{\tau_{1}, \tau_{2}, \ldots, \tau_{n-1}\right\} \neq \emptyset \text {. }
$$


Let $h(x)$ be the greatest common divisor of $P_{B}(x)$ and $P_{B(1 \mid 1)}(x)$. Then $P_{B}(x)=$ $h(x) f(x)$ and $P_{B(1 \mid 1)}(x)=h(x) g(x)$. Apply Lemma 2.3, there exists a unitary matrix $U_{1}$ in the form $\left(\begin{array}{ll}1 & 0 \\ 0 & *\end{array}\right)$, such that $U_{1} B U_{1}^{*}$ is an upper Hessenberg matrix $\hat{B}$. Due to normality of $\hat{B}$ and Lemma 2.7 ,

$$
\hat{B}=\left(\begin{array}{cc}
R & 0 \\
0 & Q
\end{array}\right)
$$

where $R$ is positively unreduced upper Hessenberg, $Q$ is normal and

$$
P_{R}(x)=f(x), P_{R(1 \mid 1)}(x)=g(x) \text {, and } P_{Q}(x)=h(x) .
$$

Notice that $f(x), g(x)$ and $h(x)$ are uniquely determined by $\sigma(B)$ and $\sigma(B(1 \mid 1))$, and $\sigma(R) \cap \sigma(R(1 \mid 1))=\emptyset$ due to how $f(x)$ and $g(x)$ are defined. $R$ is uniquely determined due to Theorem 2.5. So $\hat{B}$ is uniquely determined. Repeat the same process to $\tilde{B}$, it is not hard to see that there exists a unitary matrix $U_{2}$ in the form $\left(\begin{array}{ll}1 & 0 \\ 0 & *\end{array}\right)$ such that $U_{2} \tilde{B} U_{2}^{*}$ is $\hat{B}$ equals $\hat{B}=\left(\begin{array}{cc}R & 0 \\ 0 & \hat{Q}\end{array}\right)$, where $P_{\hat{Q}}(x)=h(x)$. As $Q$ and $\hat{Q}$ are normal with the same eigenvalues, they are unitarily similar and the result follows.

\section{Hermitian Case}

In this section we specify Theorem 1.1 for the case of real $\lambda_{i}, \mu_{i}$ and $\nu_{i}$ bringing us back to the classical A. Horn setting, as normal matrices with real eigenvalues are Hermitian. The fact that the prescribed numbers are real give that conditions (1.4) and (1.5) can be reduced to interlacing inequalities. We arrange real $n$ tuples in non-increasing order; for instance $\mu_{1} \geq \mu_{2} \geq \ldots \geq \mu_{n}$. In the case when $A$ has rank 1, the Horn inequalities [5] simplify significantly; see below. As in the previous section, we set $\lambda_{2}=\lambda_{3}=\cdots=\lambda_{n}=0$, which by our non-increasing convention then implies that $\lambda_{1}>0$.

Theorem 3.1. Let non-increasingly ordered tuples $\lambda=\left(\lambda_{1}, 0, \ldots, 0\right), \mu=\left(\mu_{1}, \mu_{2}\right.$, $\left.\ldots, \mu_{n}\right), \nu=\left(\nu_{1}, \nu_{2}, \ldots, \nu_{n}\right) \in \mathbb{R}^{n}$ be given. There exists Hermitian matrices $A, B$ such that

$$
\sigma(A)=\lambda, \quad \sigma(B)=\mu, \quad \sigma(A+B)=\nu
$$

if and only if the trace equality (1.2) and one (and consequently, both) of the following hold:

(i) The Horn inequalities hold, i.e.,

$$
\begin{cases}\max \left\{\lambda_{1}+\mu_{n}, \mu_{1}\right\} \leq \nu_{1} \leq \lambda_{1}+\mu_{1} & \\ \mu_{k} \leq \nu_{k} \leq \min \left\{\lambda_{1}+\mu_{k}, \mu_{k-1}\right\}, & k=2, \ldots, n, \\ \sum_{i=1}^{k} \nu_{i} \leq \lambda_{1}+\sum_{i=1}^{k} \mu_{i}, & k=2, \ldots, n .\end{cases}
$$

(ii) $\nu_{1} \geq \mu_{1} \geq \ldots \geq \nu_{n} \geq \mu_{n}$.

Moreover, if $\tilde{A}, \tilde{B}$ is another solution, then there exists a unitary matrix $U$, so that

$$
A=U \tilde{A} U^{*}, \quad B=U \tilde{B} U^{*} .
$$


Proof. Using that $\lambda_{1}>0$ and that the $\mu$ 's and $\nu$ 's are real, it is not hard to see that (ii) is equivalent to the pair of conditions (1.4) and (1.5). This gives the if and only if result using condition (ii).

It now remains to show that (i) is equivalent to (ii), under the assumption that trace equality (1.2) holds.

Let $\mu$ and $\nu$ satisfy the interlacing condition (ii). Then

$$
\lambda_{1}=\sum_{i=1}^{n} \nu_{i}-\sum_{i=1}^{n} \mu_{i}=\nu_{1}-\mu_{n}+\sum_{i=1}^{n-1}\left(\nu_{i+1}-\mu_{i}\right) \leq \nu_{1}-\mu_{n} .
$$

Next, for $k=1, \ldots, n$,

$$
\nu_{k}-\left(\lambda_{1}+\mu_{k}\right)=\sum_{i=1, i \neq k}^{n}\left(\mu_{i}-\nu_{i}\right) \leq 0 .
$$

Lastly,

$$
\lambda_{1}+\sum_{i=1}^{k} \mu_{k}-\sum_{i=1}^{k} \nu_{i}=\sum_{i=k+1}^{n}\left(\nu_{i}-\mu_{i}\right) \geq 0
$$

Thus (i) holds.

That (i) implies (ii) is trivial (we actually do not need the trace equality here).

The uniticity up to unitary similarity follows directly from Theorem 1.1.

In [2] the uniticity in Theorem 3.1 was proven by using a result by Hochstadt [4], which gives uniqueness of a tridiagonal Hermitian matrix with prescribed different eigenvalues for the full matrix and its principal submatrix of one size smaller. The uniqueness for the full Horn problem is addressed in a corollary in the appendix of [8], where the result is stated in terms of honeycombs.

Acknowledgement. Both authors were partially supported by NSF grant DMS-0901628.

\section{REFERENCES}

1. T. Ando and R. Bhatia, Eigenvalue inequalities associated with the Cartesian decomposition, Linear Multilinear Algebra 22 (1987), no. 2, 133-147.

2. Lei Cao, A new formulation and uniqueness of solutions to A. Horn's problem, Dissertation, 2012.

3. G.H. Golub and C.F. Van Loan. Matrix Computations, Johns Hopkins Studies in the Mathematical Sciences, Johns Hopkins University Press, Baltimore, MD, fourth edition, 2013.

4. H. Hochstadt, On the construction of a Jacobi matrix from spectral data, Linear Algebra Appl. 8 (1974), 435-446.

5. A. Horn, Eigenvalues of sums of Hermitian matrices, Pacific J. Math. 12 (1962), 225-241.

6. A.A. Klyachko, Stable bundles, representation theory and Hermitian operators, Selecta Math. (N.S.) 4 (1998), no. 3, 419-445.

7. A. Knutson and T. Tao, The honeycomb model of $\mathrm{GL}_{n}(\mathbf{C})$ tensor products. I. Proof of the saturation conjecture, J. Amer. Math. Soc. 12 (1999), no. 4, 1055-1090.

8. A. Knutson, T. Tao and C. Woodward, The honeycomb model of $\mathrm{GL}_{n}(\mathbb{C})$ tensor products. II. Puzzles determine facets of the Littlewood-Richardson cone, J. Amer. Math. Soc. 17 (2004), no. 1, 19-48 (electronic). 
9. C.-K. Li, Y.-T. Poon and N.-S. Sze, Eigenvalues of the sum of matrices from unitary similarity orbits, SIAM J. Matrix Anal. Appl. 30 (2008), no. 2, 560-581.

10. S.M. Malamud, Inverse spectral problem for normal matrices and the Gauss-Lucas theorem, Trans. Amer. Math. Soc. 357 (2005), no. 10, 4043-4064.

11. H. Wielandt, On eigenvalues of sums of normal matrices, Pacific J. Math. 5 (1955), 633638.

Department of Mathematics, Drexel University, Philadelphia, PA, 19104, USA.

E-mail address: c1428@drexel.edu

E-mail address: hugo@math.drexel.edu 